\title{
Reaction time in hypothyroid and hyperthyroid patients before and after drug treatment
}

\author{
Shah $\mathrm{SH}^{1}$, Nahar $\mathrm{PS}^{2}$ \\ ${ }^{1}$ Assistant Professor, Department of Physiology, B J Medical College, Pune. \\ ${ }^{2}$ Assistant Professor, Department of Physiology, B J Medical College, Pune
}

\begin{abstract}
Reaction time (RT) is the time interval between onset of stimulus and occurrence of response. There are few studies on audiovisual RT in patients of thyroid dysfunction and effect of treatment on RT. Hence this study was aimed to compare the audiovisual RT in hypothyroid and hyperthyroid patients with age matched normal healthy controls. We also studied that whether there is any improvement in RT after giving treatment to these patients with L- Thyroxine and Carbimazole respectively after 21 days. RT was measured by Response analyser, a simple machine manufactured locally .This machine provide audio ( low and high ) and visual ( red, green and yellow colours each of low and high intensity ). RT was measured in 20 hypothyroid , 20 hyperthyroid and 20 healthy controls. Again RT was measured after giving treatment with LThyroxine and Carbimazole respectively after 21 days. Statistical analysis was done by using Student's t test. The results showed that there was a significant increase in audio-visual RT in hypo as well as hyperthyroid patients as compared to the control group. In hypothyroid and hyperthyroid patients the auditory RT decreased significantly after treatment $(\mathrm{p}<0.05)$. Hence it appears that measurement of auditory RT is probably a sensitive indicator of thyroid dysfunction, in absence of any other clinical pathology.
\end{abstract}

Keywords: reaction time, hypothyroidism, hyperthyroidism

\section{Introduction}

Reaction time is the time interval between onset of stimulus and occurrence of response. It is an important parameter representing the integration of sensory, motor and coordination system of the body. This parameter is widely used in selection of aeroplane pilots, car drivers and loco drivers. It is also used in industrial situation to prevent accidents. ${ }^{1,2)}$

However this parameter has not been studied in patients of thyroid dysfunction. Since the thyroid hormones are involved in metabolic activities of almost all the tissues, reaction time is expected to be altered in these group of patients. Hence in this study auditory and visual reaction time is studied in hypothyroid and hyperthyroid patients before and after drug treatment. ${ }^{(3)}$

\section{Aims and objectives}

1. To find out reaction time in hypothyroid patients before and after treatment with L- thyroxine.

2. To find out reaction time in hyperthyroid patients before and after treatment with carbimazole.

\section{Materials and methods}

The study was conducted on adult patients of hypothyroidism and hyperthyroidism attending the endocrinology OPD of the institute along with the normal human beings as the controls ( age group $30-50 \mathrm{yrs}$ )

Following three groups were included in the study

1. Controls : 20 Normal healthy volunteers

2. Group I : 20 freshly diagnosed cases of primary hypothyroidism

3. Group II : 20 freshly diagnosed cases of primary hyperthyroidism

\section{Recording of reaction time ${ }^{(4)}$}

Reaction time was measured by Response analyser, a simple machine manufactured locally. This machine provide audio ( low and high ) and visual ( red, green and yellow colours each of low and high intensity ) stimuli. The response is given by pressing a soft touch key with a thumb. It provides display range of $9.999 \mathrm{sec}$ with an accuracy of 1 milisecond.

The reaction time was recorded in an isolated, air conditioned, adequately illuminated, noise free room between $10-11 \mathrm{am}$. On each visit it was ensured that the location and direction of instrument as well as the subject is constant, so also the distance between them.

The reaction time is measured as follow:

1. A ready signal was given to the subject

2. Subject was asked to press the button as soon as he receives the stimulus

3. Reaction time was displayed in milliseconds

4. Five readings were taken for each stimulus by randomly varying the foreperiod ( range $0-10 \mathrm{sec}$ ) 
5. The mean of the above readings was taken as reaction time for that stimulus, on a particular visit.

The subjects were assessed as mention above at the time of diagnosis ( Visit 1) and after the study period of 21 days ( Visit 2)

During this period

1. Control group : did not receive any medication

2. Hypothyroid group ( Group I ) : received L - thyroxine ( Eltroxine ) $0.1 \mathrm{mg}$ daily between 8-9 am on empty stomach for 21 days

3. Hyperthyroid group ( Group II ) : received Carbimazole ( Neomercazole ) $5 \mathrm{mg}$ four times daily for 21 days

It was ensured that none of the subject in any group will receive any other medication during the study period, and did not have any other concomitant disease.

Statistical analysis : Student's unpaired t test was applied to compare the audio-visual RT between the controls and hypothyroid as well as hyperthyroid patients. Paired t test was applied to compare the RT before and after treatment in hypo and hyperthyroid patients.

\section{Results}

There was a significant increase in audio-visual reaction time in hypo as well as hyperthyroid patients as compared to the control group. In hypothyroid patients the auditory reaction time decreased significantly after treatment with thyroxine $(\mathrm{p}<$ 0.05 ). Similarly in hyperthyroid patients the auditory time decreased significantly after treatment with carbimazole $(\mathrm{p}<0.05$ ). Both the groups also showed decrease in visual reaction time but it was not statistically significant. $(\mathrm{p}>0.05)$.

Table 1: Reaction time for various stimuli in different group at first visit ( V 1 )

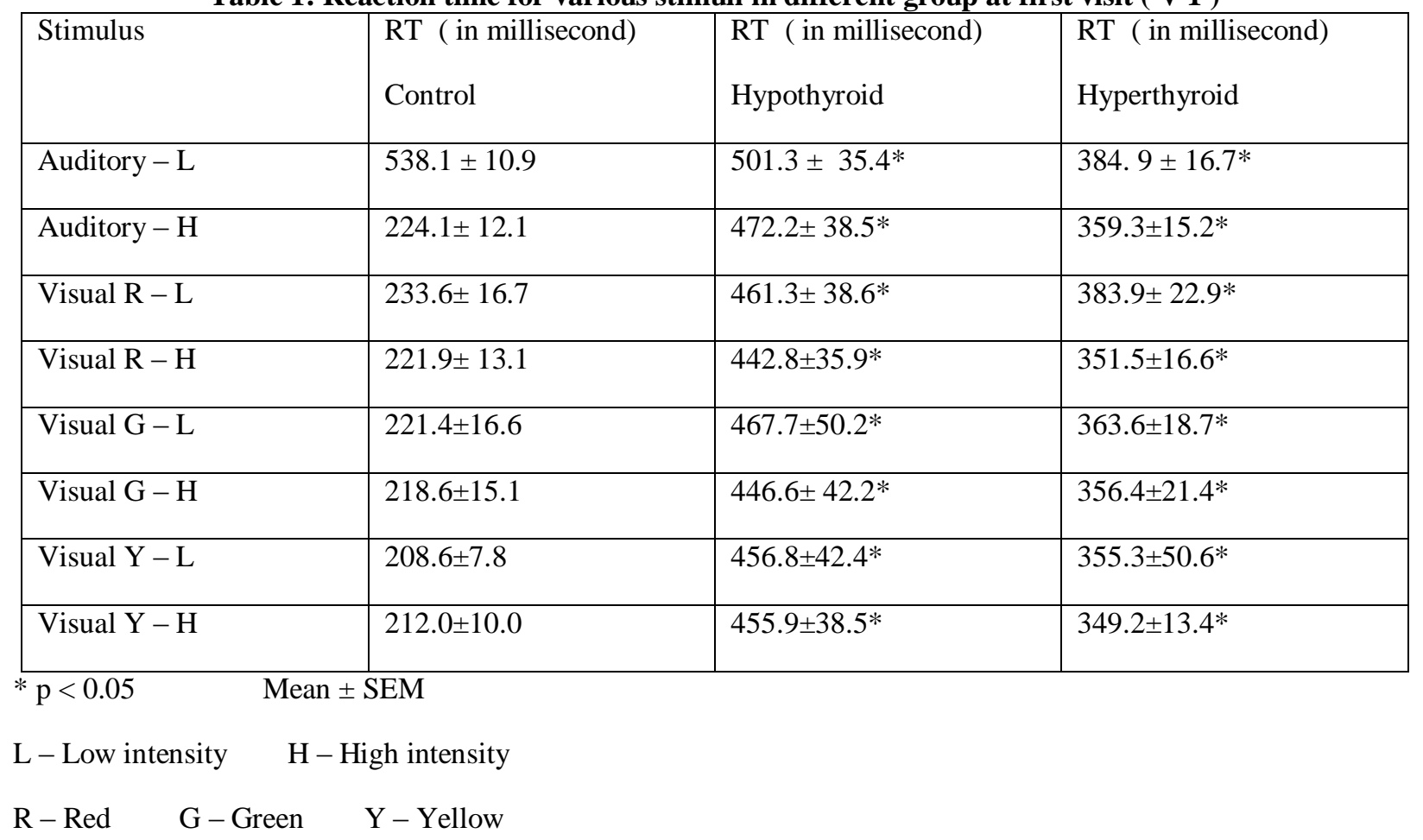


Table 2: Reaction time for various stimuli in hypothyroid group before( $V 1$ ) and after treatment with $L$ - thyroxine ( V 2 )

\begin{tabular}{|c|c|c|}
\hline Stimulus & $\begin{array}{l}\text { RT ( in millisecond) } \\
\text { V } 1\end{array}$ & $\begin{array}{l}\text { RT ( in millisecond) } \\
\text { V } 2\end{array}$ \\
\hline Auditory - L & $501.3 \pm 35.4$ & $408.9 \pm 24.6^{*}$ \\
\hline Auditory $-\mathrm{H}$ & $472.2 \pm 38.5$ & $383.9 \pm 26.0^{*}$ \\
\hline Visual R - L & $461.1 \pm 38.6$ & $423.0 \pm 32.0$ \\
\hline Visual $\mathrm{R}-\mathrm{H}$ & $442.8 \pm 35.9$ & $405.2 \pm 28.5$ \\
\hline Visual G-L & $467.7 \pm 5.2$ & $406.6 \pm 25.5$ \\
\hline Visual $\mathrm{G}-\mathrm{H}$ & $446.0 \pm 42.2$ & $387.7 \pm 20.6$ \\
\hline Visual Y - L & $456.8 \pm 42.4$ & $420.8 \pm 27.6$ \\
\hline Visual $\mathrm{Y}-\mathrm{H}$ & $455.9 \pm 38.5$ & $408.4 \pm 25.9$ \\
\hline
\end{tabular}

L - Low intensity $\quad \mathrm{H}-$ High intensity

$\mathrm{R}-$ Red $\quad \mathrm{G}-$ Green $\quad \mathrm{Y}-$ Yellow

Table 3: Reaction time for various stimuli in hyperthyroid group before( $\mathrm{V} 1$ ) and after treatment with carbimazole ( V 2)

\begin{tabular}{|l|l|l|}
\hline Stimulus & RT (in millisecond) & RT ( in millisecond) \\
& V 1 & V 2 \\
\hline Auditory - L & $384.9 \pm 16.7$ & $362.6 \pm 30.5$ \\
\hline Auditory - H & $359.3 \pm 15.2$ & $310.5 \pm 8.5 *$ \\
\hline Visual R - L & $383.9 \pm 22.9$ & $347.9 \pm 23.3$ \\
\hline Visual R - H & $351.5 \pm 16.6$ & $321.1 \pm 18.4$ \\
\hline Visual G - L & $363.6 \pm 18.7$ & $318.0 \pm 12.7$ \\
\hline Visual G - H & $356.4 \pm 21.4$ & $312.8 \pm 14.7$ \\
\hline Visual Y - L & $355.3 \pm 50.6$ & $315.7 \pm 18.4$ \\
\hline Visual Y - H & $349.2 \pm 13.4$ & $304.2 \pm 17.1$ \\
\hline
\end{tabular}

$* \mathrm{p}<0.001 \quad$ Mean \pm SEM

L - Low intensity $\quad \mathrm{H}-$ High intensity

$\mathrm{R}-$ Red $\quad \mathrm{G}-$ Green $\quad \mathrm{Y}-$ Yellow 


\section{Discussion}

In our study we found significant increase in audio-visual reaction time in hypo as well as hyperthyroid patients as compared to the control group. In hypothyroid patients the auditory reaction time decreased significantly after treatment with thyroxine $(\mathrm{p}<0.05)$. Similarly in hyperthyroid patients the auditory time decreased significantly after treatment with carbimazole $(\mathrm{p}<$ 0.05 ). Both the groups also showed decrease in visual reaction time but it was not statistically significant $(p>0.05)$.

In hypothyroid group, both audio-visual reaction time ( RT ) were significantly higher than the control group. This is probably because of generalised decrease in metabolic rates affecting the sensory receptors, neural pathways and skeletal muscles. After administration of L-thyroxine, only auditory RT showed significant reduction. This is probably because of higher dependency of auditory receptors or their metabolic rates on serum T3 or T4.

In hyperthyroid group, interestingly both audio-visual RT were significantly higher than the control group. After treatment with carbimazole only the auditory RT showed significant decreased. It seems that optimum level of thyroid hormone, i. e. maintainance of euthyroid status is very much essential as far as the auditory reaction time is concern, derangement in either direction ( hypothyroidism or hyperthyroidism ) will hamper the normal functioning of auditory mechanism. ${ }^{(5,6)}$

\section{Conclusion}

The audiovisual RT was significantly increased in patients of primary hypothyroidiam and hyperthyroidism. Administration of $L$ thyroxine and carbimazole respectively, leads to significant decrease in auditory reaction time within 3 weeks

There is also decrease in visual reaction time but it was not statistically significant which needs further research.

Hence it appears that measurement of auditory reaction time which is very simple procedure may be a sensitive indicator of thyroid dysfunction, in absence of any other clinical pathology.

\section{References}

1. Lally N, Nettlbeck T. Intelligance, reaction time and inspection time. American J of Mental Deficiency. 1977; 82: 273-81.

2. Mathur LA, Tendon HB. Reaction time experiments. Journal of abnormal psychology. 1964;72:35-40.

3. Kaplan M. Symposium on thyroid diseases. The Medical Clinic of North America. 1985;5:849-1005.

4. Jensen AR. Learning and Reaction time - Educability and Group differences. Harper and Row. New York $1973: 69-90$.

5. Boumeister AA, Kellas G. Distribution of reaction time of retardates and normals. American J of Mental Deficiency. 1968;72: 715-8.

6. Boumeister AA, Kellas G. Intrasubject response variability in relation to intelligence. J of Abnormal Psychology. 1968; 73;421-23. 